\title{
HETTER
}

\section{Suboptimum coherent detection of orthogonal phase-modulated signals}

\author{
Horng-Shyang Liaw ${ }^{\mathrm{a}, *}$, Hung-Tai Lin ${ }^{\mathrm{b}}$, Char-Dir Chung ${ }^{\mathrm{c}}$ \\ ${ }^{a}$ Department of Electrical Engineering, National Central University, Chung-Li, Taiwan 320, ROC \\ ${ }^{\mathrm{b}}$ Compal Communications, Inc., Taipei 105, Taiwan, ROC \\ ${ }^{\mathrm{c}}$ Department of Electrical Engineering and Graduate Institute of Communication Engineering, National Taiwan University, \\ Taipei, Taiwan 106, ROC
}

Received 21 June 2006; received in revised form 13 November 2006

\begin{abstract}
A suboptimum scheme for coherent demodulation of orthogonal phase-modulated signals in the additive white Gaussian noise channel is proposed and analyzed in terms of bit error probability. It is analytically shown that the suboptimum scheme provides the bit error performance competitive to the conventional optimum scheme, while requiring much less implementation complexity.
\end{abstract}

(C) 2006 Elsevier GmbH. All rights reserved.

Keywords: Orthogonal phase modulation; Coherent detection; Phase shift keying; Orthogonal group signaling

\section{Introduction}

Orthogonal phase modulation (OPM) is a multidimensional linear modulation format defined by combining the techniques of orthogonal group signaling and phase-shift keying [1]. Specifically, when constructed from a basis set of $N_{c}$ orthonormal basis signals, the OPM signal is composed at each signaling time by grouping $L$ phase-shift keyed (PSK) basis signals, in which the $L$ basis signals are chosen from distinct basis signal groups according to a common grouping data symbol and independently phase-shift keyed in a $K$-ary alphabet. For convenience, this signaling format is dubbed herein by $\left(N_{c}, L, K\right)$ OPM. By orthogonally multiplexing spectrally overlapping OPM signals, the orthogonally multiplexed OPM family proposed in [1] can provide a great choice of modulations giving various error and spectral performance characteristics. The optimum scheme for coherently demodulating the OPM signals in the additive

\footnotetext{
* Corresponding author. Tel.: +88634245488; fax: +88634244920.

E-mail addresses: liaw@cht.com.tw (H.-S. Liaw), hungtai_lin@compal.com (H.-T. Lin), cdchung@cc.ee.ntu.edu.tw (C.-D. Chung).
}

white Gaussian noise (AWGN) channel was also developed therein and shown to enable digital realization with a complexity of order $K N_{c}$ operations required at each signaling time.

In the letter, we propose a simple two-stage suboptimum decision scheme which requires less digital realization complexity than the optimum scheme. It is analytically shown that the suboptimum scheme can provide the bit error probability (BEP) characteristics very close to the optimum one for most of $\left(N_{c}, L, K\right)$ OPMs while being simpler to realize.

\section{Signal model}

Let $\Psi_{k}=\left[a_{k}, \mathbf{b}_{k}\right]$ be the data supersymbol transmitted at the $k$ th signaling time, where $a_{k} \in \mathscr{Z}_{N_{c} / L}$ is the grouping data symbol, $\mathbf{b}_{k}=\left(b_{k}^{(0)}, b_{k}^{(1)}, \ldots, b_{k}^{(L-1)}\right)$ is the vector of $L$ PSK data symbols with $b_{k}^{(l)} \in \mathscr{Z}_{K}$, with $\mathscr{Z}_{K} \triangleq$ $\{0,1, \ldots, K-1\}$, and all data symbols are generated independently and take value with equal probability from respective alphabets. The modulation parameters $N_{c}, L$ and $K$ are assumed to be integer powers of two with $N_{c} \geqslant 2,1 \leqslant L \leqslant N_{c}$, 
and $K \geqslant 2$. Let $\Omega \triangleq\left\{p_{n}(t) \cos \left(\omega_{n} t\right), p_{n}(t) \sin \left(\omega_{n} t\right)\right\}_{n=0}^{N c-1}$ denote the underlined set of $2 N_{c}$ real-valued basis signals that are mutually orthonormal and also orthonormal to the $l T$ shifted versions of all basis signals, where $T$ is the signaling time for one supersymbol, $p_{n}(t)$ represents the common pulse accompanying the quadrature carriers with frequency $\omega_{n}$, and $\omega_{n}$ 's are noncoherently orthogonally spaced with $\omega_{n}-\omega_{m}=2 \pi(n-m) / T$. Based on $\Omega$, the unit-power OPM signal can be generally formulated as

$$
\begin{aligned}
s(t) \triangleq & \sqrt{\frac{T}{L}} \sum_{k} \sum_{l=0}^{L-1} p_{\frac{l N_{c}}{L}+a_{k}}(t-k T) \\
& \times \cos \left(\omega_{\frac{l N_{C}}{L}+a_{k}}(t-k T)+\frac{2 \pi}{K} b_{k}^{(l)}\right) .
\end{aligned}
$$

In constructing $s(t)$ for the $k$ th signaling time, a group of $L$ pairs of quadrature pulsed carriers is selected from $\Omega$ according to the $N c / L$-ary symbol $a_{k}$, and these $L$ carrier pairs are individually phase-shift-keyed with $L K$-ary phase symbols $b_{k}^{(l)}$ 's. By signaling so, $s(t)$ carries $\lambda_{a}+\lambda_{b}$ bits per $T$ seconds with $\lambda_{a} \triangleq \log _{2}\left(N_{c} / L\right)$ and $\lambda_{b} \triangleq L \log _{2} K$ denoting the numbers of information bits represented by $a_{k}$ and $\mathbf{b}_{k}$, respectively.

\section{Suboptimum OPM decision}

Considering the coherent demodulation of the OPM signal in the AWGN channel and assuming all the basis signals are perfectly synchronized, the receiver correlates the received signal $r(t)$ with local reference basis signals to yield a set of sufficient statistics $\left\{R_{n, k}^{(c)}, R_{n, k}^{(s)}\right\}_{n=0}^{N c-1}$ for the $k$ th signaling time, with $R_{n, k}^{(c)}=\int_{-\infty}^{\infty} r(t) p_{n}(t-k T) \cos \left(\omega_{n}(t-k T)\right) \mathrm{d} t$ and $R_{n, k}^{(s)}=\int_{-\infty}^{\infty} r(t) p_{n}(t-k T) \sin \left(\omega_{n}(t-k T)\right) \mathrm{d} t$. Operating on $\left\{R_{n, k}^{(c)}, R_{n, k}^{(s)}\right\}_{n=0}^{N c-1}$, the proposed suboptimum scheme makes decision on $a_{k}$ and $\mathbf{b}_{k}$ in two separate steps. At the first step, the decision on $a_{k}$ is made by choosing the largest sum of squared envelopes among $\left\{\Lambda_{a_{k}} ; a_{k} \in \mathscr{Z}_{N_{c} / L}\right\}$ with

$\Lambda_{a_{k}}=\sum_{l=0}^{L-1}\left\{\left(R_{\frac{l N_{c}}{L}+a_{k}, k}^{(c)}\right)^{2}+\left(R_{\frac{l N_{c}}{L}+a_{k}, k}^{(s)}\right)^{2}\right\}$

and thus the decision $\widehat{a}_{k}$ is given by $\widehat{a}_{k}=\arg \max _{a_{k}} \Lambda_{a_{k}}$. With the decision $\widehat{a}_{k}$, the decision on $\mathbf{b}_{k}$ follows the conventional PSK decision scheme [2] and is given at the second step by

$$
\begin{aligned}
\widehat{b}_{k}^{(l)}= & \arg \max _{b_{k}^{(l)}}\left\{\cos \left(\frac{2 \pi}{K} b_{k}^{(l)}\right) \cdot R_{\frac{l N_{c}}{L}+\widehat{a}_{k}, k}^{(c)}\right. \\
& \left.-\sin \left(\frac{2 \pi}{K} b_{k}^{(l)}\right) \cdot R_{\frac{l N_{c}}{L}+\widehat{a}_{k}, k}^{(s)}\right\}
\end{aligned}
$$

for each $l \in\{0,1, \ldots, L-1\}$.

When digitally realized, the proposed two-step scheme requires a complexity of order $L K+N_{c}$ operations at each
Table 1. Comparison of digital realization complexity between optimum and suboptimum coherent decision schemes

\begin{tabular}{lll}
\hline $\begin{array}{l}\text { Number of operations } \\
\text { required per } T \text { seconds }\end{array}$ & $\begin{array}{l}\text { Optimum } \\
\text { scheme }\end{array}$ & $\begin{array}{l}\text { Suboptimum } \\
\text { scheme }\end{array}$ \\
\hline $\begin{array}{l}\text { Real multiplications } \\
\text { Maximizations }\end{array}$ & $2 K N_{c}$ & $2\left(L K+N_{c}\right)$ \\
Real additions & $K N_{c}$ & $L K+N_{c} / L$ \\
$K N_{c}$ & $L K+N_{c}$ \\
\hline
\end{tabular}

signaling time. Table 1 compares the realization complexity required by optimum and suboptimum decision schemes. When $K$ (phase alphabet size) or $2 N_{c}$ (signal space dimensionality) is large, noticeable complexity reduction over the optimum scheme can be achieved by the proposed scheme.

Consider the BEP performance of the proposed scheme assuming that Gray mapping of bits to symbols is used. First, we evaluate the probability of making a correct decision at the first step, denoted by $P_{c a}$. Because all $a_{k}$ values are transmitted equally likely, $P_{c a}$ is the probability that $\Lambda_{i}$ is the largest measurement among $\Lambda_{j}$ 's for $j \in$ $\mathscr{Z}_{N_{c} / L}$ when a particular $a_{k}=i$ is transmitted. Since $\Lambda_{j}$ 's are statistically independent given $a_{k}=i, P_{c a}$ is given by $P_{c a}=\int_{0}^{\infty}\left[\int_{0}^{\Lambda_{i}} f_{\Lambda_{n}}\left(\Lambda_{n}\right) \mathrm{d} \Lambda_{n}\right]^{\frac{N_{c}}{L}-1} f_{\Lambda_{i}}\left(\Lambda_{i}\right) \mathrm{d} \Lambda_{i}$ for some $n \neq$ $i$, where $f_{\Lambda_{n}}\left(\Lambda_{n}\right)$ and $f_{\Lambda_{i}}\left(\Lambda_{i}\right)$ are central and noncentral chi-square probability density functions with $2 L$ degrees of freedom, respectively. By quoting [2, Eq. (2-1-138)] and [2, Eq. (2-1-146)], this integral can be simplified to

$$
\begin{aligned}
P_{c a}= & \sum_{\substack{m_{0}+m_{1}+\cdots+m_{L}=\frac{N_{c}}{L}-1 \\
m_{i} \in \mathscr{Z}_{N_{c} / L}}}\left(\begin{array}{c}
\frac{N_{c}}{L}-1 \\
m_{0}, m_{1}, \ldots, m_{L}
\end{array}\right) \\
& \times \frac{\left(L-1+\sum_{i=1}^{L-1} \mathrm{i} m_{i+1}\right) ! \cdot \mathrm{e}^{-\left(\lambda_{a}+\lambda_{b}\right) \gamma_{b}}}{(-1)^{\left(m_{0}-\frac{N_{c}}{L}+1\right)}(L-1) !} \\
& \times \frac{\Phi\left(L+\sum_{i=1}^{L-1} \mathrm{i} m_{i+1}, L ; \frac{\left(\lambda_{a}+\lambda_{b}\right) \gamma_{b}}{\left(N_{c} / L\right)-m_{0}}\right)}{\left(\frac{N_{c}}{L}-m_{0}\right)^{\left(L+\sum_{i=1}^{L-1} \mathrm{i} m_{i+1}\right)} \prod_{i=1}^{L-1}(i !)^{m_{i+1}}} .
\end{aligned}
$$

In Eq. (2), $\gamma_{b}$ is the ratio of received signal energy per bit to noise power spectral density level and $\Phi(\alpha, \beta ; x)$ is the confluent hypergeometric function as defined in $[2, \mathrm{Eq}$. (2-1-134)].

When $\widehat{a}_{k}$ is correctly decided, the average number of bit errors on decision $\widehat{\mathbf{b}}_{k}$ is given by $P_{c a} P_{e b} \lambda_{b}$ where $P_{e b}$ represents the BEP of the conventional PSK decision scheme and can be evaluated based on conventional approaches, e.g., [3]. When $\widehat{a}_{k}$ is incorrectly decided, the average numbers of bit errors on decisions $\widehat{a}_{k}$ and $\widehat{\mathbf{b}}_{k}$ are given by

$$
\begin{aligned}
& \sum_{n=1}^{\lambda_{a}} n\left(\begin{array}{c}
\lambda_{a} \\
n
\end{array}\right) \frac{\left(1-P_{c a}\right)}{\left(N_{c} / L\right)-1}=\lambda_{a} \frac{N_{c}}{2 L}\left[\frac{1-P_{c a}}{\left(N_{c} / L\right)-1}\right] \text { and } \\
& \frac{\left(1-P_{c a}\right)}{2} \lambda_{b},
\end{aligned}
$$


respectively, where $\frac{\left(1-P_{c a}\right)}{\left(N_{c} / L\right)-1}$ is the probability that any symbol other than the correct one is chosen as the decision $\widehat{a}_{k}$. Combing both cases, the average BEP of the suboptimum scheme for $\left(N_{c}, L, K\right)$ OPM is derived as

$P_{b}=\frac{P_{c a} P_{e b} \lambda_{b}+\frac{1}{2}\left(1-P_{c a}\right)\left\{\left[\frac{N_{c} / L}{\left(N_{c} / L\right)-1}\right] \lambda_{a}+\lambda_{b}\right\}}{\lambda_{a}+\lambda_{b}}$.

\section{Bit error probability results}

Figs. 1 and 2 illustrate the BEP characteristics of optimum and suboptimum OPM decision schemes with $L=2$.

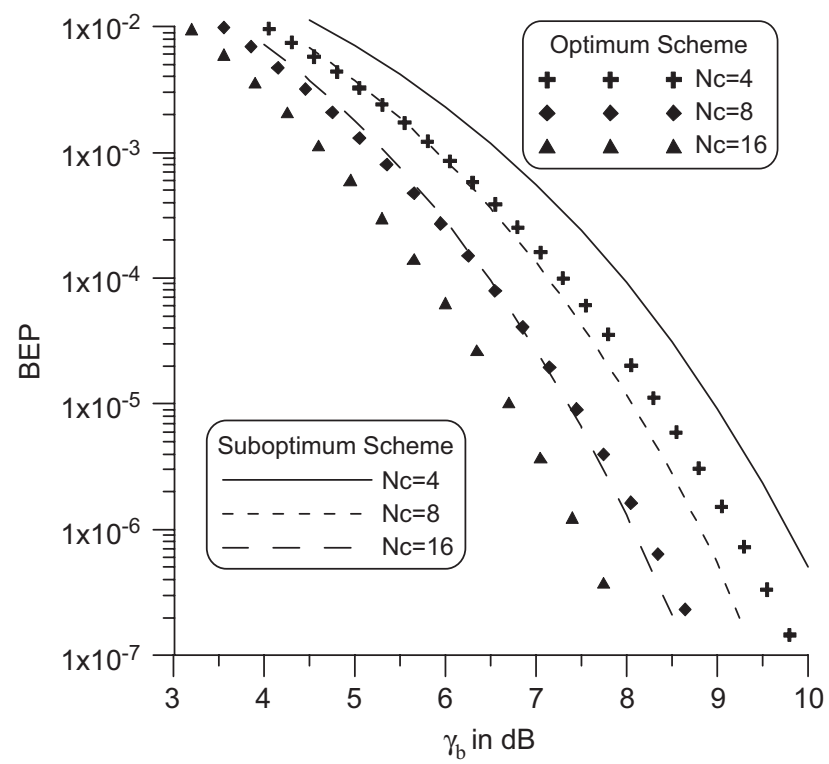

Fig. 1. BEP comparison of optimum and suboptimum $\left(N_{c}, 2,2\right)$ OPM decision schemes.

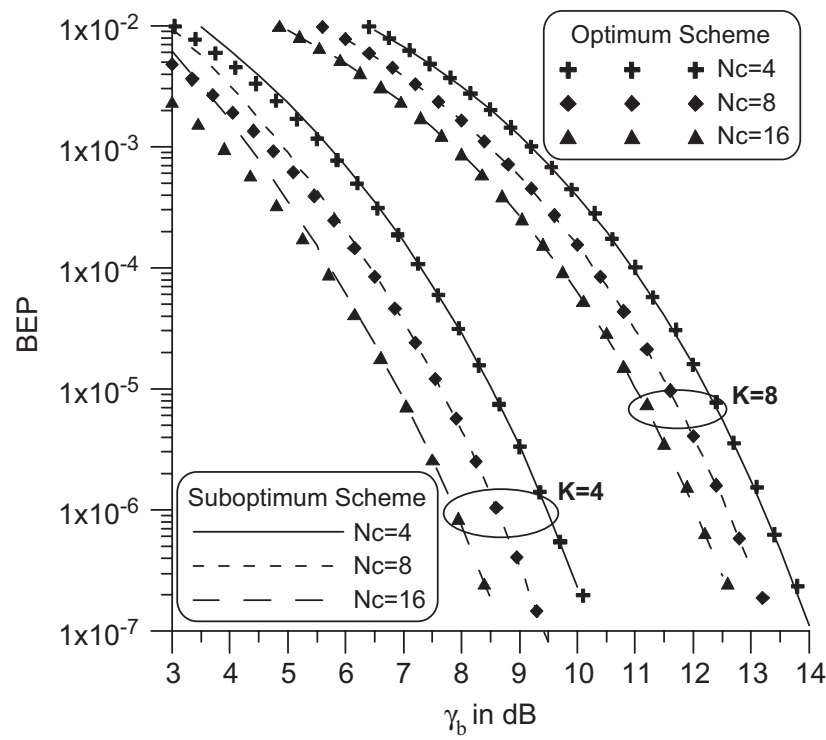

Fig. 2. BEP comparison of optimum and suboptimum $\left(N_{c}, 2, K\right)$ OPM decision schemes with $K=4$ and 8 .
In Fig. 1, the suboptimum scheme with $K=2$ is shown to degrade from the corresponding optimum scheme by about $0.6 \mathrm{~dB}$ and the degradation remains the same for different $N_{c}$ values. In Fig. 2, the suboptimum scheme with $K=4$ and 8 are shown to perform very close to the corresponding optimum scheme. It is also found by the authors that the BEP of the suboptimum scheme is dominated by the decision on $a_{k}$ when $K=2$ and the decision on $\mathbf{b}_{k}$ when $K \geqslant 4$. Note that the above performance trends also hold for other larger $L$ values.

\section{Conclusion}

A suboptimum decision scheme for coherently demodulating the OPM signals is proposed in the letter. The suboptimum scheme is shown to perform in BEP very close to the optimum scheme when $K \geqslant 4$. When the phase alphabet size or the signal space dimensionality is large, the suboptimum scheme is found to require much less complexity in digital realization than the optimum scheme.

\section{References}

[1] Chung CD. Coherent and differentially coherent detections of orthogonally multiplexed orthogonal phase-modulated signals. IEEE Trans Commun 2003;51:428-40.

[2] Proakis JG. Digital communications. New York: McGrawHill; 1995.

[3] Lassing J, Ström EG, Agrell E, Ottosson T. Computation of the exact bit-error rate of coherent $M$-ary PSK with gray code bit mapping. IEEE Trans Commun 2003;51:1758-60.

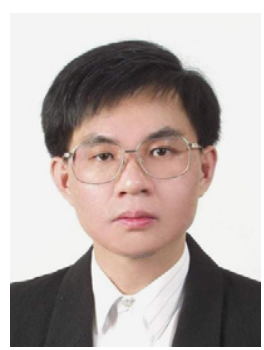

Horng-Shyang Liaw received his B.S.E.E. and M.S.E.E. degrees from the National Central University, Chung-Li, Taiwan, ROC, in 1992 and 1994, respectively. He is currently working toward the Ph.D. degree in Electrical Engineering at the National Central University. In 1994, he joined the Telecommunication Laboratories of the Chunghwa Telecom Corporation, Yang-Mei, Taiwan, where he is involved in the radio access network planning and optimization in the $3 \mathrm{G}$ mobile networks. His current research interests are in digital communications and mobile communications.

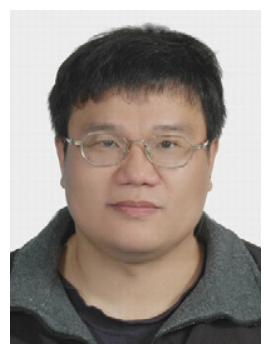

Hung-Tai Lin received B.S.E.E. degrees from the National Taipei University of Technology, Taipei, Taiwan, ROC, in 1998 and M.S.E.E. degree from the National Central University, Chung-Li, Taiwan, ROC, in 2000. In 2002, he joined the Compal Communications, Inc., Taipei, Taiwan, where he is now involved in the area of the $3 \mathrm{G}$ mobile networks protocol. 


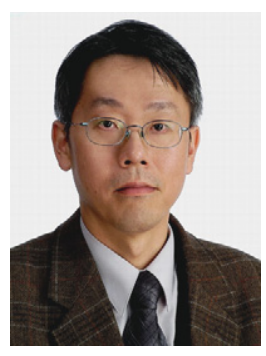

Char-Dir Chung received his B.S. degree in Electrical Engineering from the National Taiwan University, Taipei, in 1983, and M.S. and Ph.D. degrees in Electrical Engineering from the University of Southern California, Los Angeles, CA, in 1986 and 1989, respectively. From 1989 to 1992 he was with the LinCom Corporation, Los Angeles. From 1992 to 2005 he was with the National
Central University, Chung-Li, Taiwan, ROC, where he was Professor and Head of the Communication Engineering Department. Since 2005, he has been on the faculty at Department of Electrical Engineering and the Graduate Institute of Communication Engineering, National Taiwan University, Taipei, Taiwan. His current research interests are in digital modulation theory, satellite communication, spread spectrum communication, and wireless communication. He has published more than 40 journal and conference papers and holds two patents in these areas. 